\title{
EFFECT OF CLEARANCE AND DISPLACEMENT OF ATTRACTED DISK AND ALSO OF A CERTAIN AR- RANGEMENT OF CONDUCTING HOOPS, UPON THE CONSTANT OF AN ELECTROMETER
}

\author{
By Chester Snow
}

ABSTRACT

Formulas are here derived for the force upon the attracted disk of an absolute electrometer. These take account of the clearance between attracted disk and its guard plane, their relative displacement, and the separation of the plates. The effect of using a set of conducting hoops to compensate for the finiteness of the movable plate is also computed.

\section{CONTENTS}

I. Introduction $=$

II. Effect of clearance, displacement, and separation of plates_._._. 514

III. Effect of the circular conducting hoops which are used to increase the uniformity of field at the disk _........ 527

IV. Summary _

\section{INTRODUCTION}

The density of electricity upon the surface of the attracted disk of an absolute electrometer is approximately uniform. This uniformity is ordinarily achieved (when the separation between the attracted disk and the adjustable plate is small compared to the diameter of the latter) by the use of Kelvin's guard ring in the plane of the disk and having an external diameter as large as that of the plate.

If the diameter of the plate and of the guard ring be considered infinite, then it is evident that the distribution of electricity upon the attracted disk will depend upon its small clearance with the guard ring, its small displacement below or above the plane of the latter, and the separation of the two infinite plane conductors. The approximate expression for this density of electricity is obtained and, hence, for the total force on the disk. The latter and its guard ring are at the same potential $v_{0}$ and are usually about $1 \mathrm{~cm}$ in thickness. If the clearance between the two is about $1 / 2 \mathrm{~mm}$ it is evident that very little field will penetrate this crevasse and that an exceedingly small number of lines of force will terminate upon the upper face of the disk. The distribution of electricity upon the sides of 
the disk contributes nothing directly to the downward pull upon it, but it must be taken into consideration in order to find the distribution upon the lower face of the disk. The fact that the quantity of

- electricity is negligible which would be found at a greater height than $1 \mathrm{~cm}$ upon the vertical sides of disk (or upon its upper face) enables one to simplify the electrostatic problem by assuming that the disk and its guard ring are infinitely thick. A second simplification comes from the fact that the clearance and displacement of the disk below the plane of the guard ring are small compared with the radius $r$ of the disk, which is considered to be about $5 \mathrm{~cm}$. The nonuniformity of charge will, therefore, be confined to the neighborhood of the edge of the disk, so that this distribution may be derived from the corresponding two-dimensional problem. The justification of treating the radius of the adjustable plate (and guard ring) as infinite does not lie in the fact that this radius $R$ is always large compared to the separation of the plates (for this is by no means the case for measurements contemplated at this bureau), but rather is to be found in the fact that a second type of guard conductors is used. In the case of a large absolute electrometer now nearing completion at the bureau for measuring alternating voltages up to 250,000 volts, it is necessary to use spacings as great as $110 \mathrm{~cm}$ to avoid danger of spark over. It is impracticable to make the radius $R$ so large in comparison with this spacing as to insure a sufficiently uniform flux distribution by the use of the simple guard ring only. H. B. Brooks, of this bureau, has devised the further expedient of using an additional system of guard conductors. These consist of a set of $N$ equal, concentric, equally spaced, parallel hoops (of radius $R$ ), each of which is to be kept at the potential which would be found at its position in their absence, if the two conducting plates were infinite planes. They thus compensate for the finiteness of these planes and tend to produce a more uniform charge over the face of the attracted disk. A discussion of the effect of such guard hoops seems to show that the assumption of an infinite radius for the guard plane and the adjustable plate is justified.

\section{EFFECT OF CLEARANCE, DISPLACEMENT, AND SEPARA- TION OF PLATES}

The aim of this section is to obtain an approximate formula for the electrical force acting upon a fairly thick, circular disk of about $5 \mathrm{~cm}$ radius when it hangs slightly displaced from the plane of its guard ring, and has a small clearance of the order of $1 / 2 \mathrm{~mm}$. The movable plate may be from 1 to $100 \mathrm{~cm}$ below them. The derivation of such a formula requires the solution of the electrostatic problem leading to a knowledge of the distribution of electricity over the surface of the attracted disk. 
The method here followed is that explained and illustrated in J. J. Thompson's Recent Researches in Electricity and Magnetism, third chapter. The problem here considered is somewhat more general than hitherto considered in that the lower face of the movable disk is here considered to be displaced below the plane of its guard ring, both being infinitely thick. If this displacement, $c-b$, is placed equal to zero in the solution here obtained, it reduces to the solution given by Thompson. The approximations that are made below are somewhat unusual because the separation of the plates ranges from very small to very large values.

To obtain an approximate solution we may consider first the following two-dimensional case.

Let $a, b$, and $c$ be positive reals.

Consider the complex function $z \equiv x+i y$ of the complex variable $t \equiv t_{1}+i t_{2}$ defined by

$$
\pi z=b \log t-c \log \beta+c \log \frac{t-\beta}{t-\frac{1}{\beta}}-a \alpha-i a \log \frac{t-e^{i \alpha}}{t-e^{-\mathrm{i} \alpha}}
$$

If the positive real quantities $\alpha$ and $\beta$ are so chosen that

$$
\begin{gathered}
\beta=\sqrt{\frac{a^{2}+(b+c)^{2}}{a^{2}+(b-c)^{2}}} \text { so that } 1 \leqq \beta \leqq+\infty \\
\tan \alpha=\frac{2 a b}{a^{2}-b^{2}+c^{2}} \quad 0 \leqq \alpha \leqq \pi
\end{gathered}
$$

then the derivative of (2) may be put in the form

$$
\frac{\pi}{b} \frac{d z}{d t}=\frac{\left(t^{2}-1\right)^{2}}{t(t-\beta)\left(t-\frac{1}{\beta}\right)\left(t-e^{\mathrm{i} \alpha}\right)\left(t-e^{-\mathrm{i} \alpha}\right)}
$$

This makes $\frac{d z}{d t}=0$ when $t= \pm 1$, and these points are then branch points of the multiple-valued function $z$ defined by (1). This was in fact the object in choosing $\alpha$ and $\beta$ in the manner described.

Along with $z$ we may consider a second complex function of $t$, namely $w=u+i v$ defined by

$$
w \equiv u+i v=\frac{v_{0}}{\pi} \log \left[\frac{(t-\beta)\left(t-\frac{1}{\beta}\right)}{t} \cdot \frac{\beta}{(\beta+1)^{2}}\right]
$$

To remove ambiguity, we shall specify that by the logarithms of the complex quantities occurring in (1), (4), and (5) shall be understood in every case that branch whose imaginary component vanishes when the point $t$ moves off an infinite distance to the right on the real axis of $t$. This statement, together with reference to Figure 2, will show what branch is to be understood by the inverse tangents written below. 
With this understanding we may now show that the point $z$ traces the conducting boundary of the insulating region (shown unshaded in fig. 1), when the point $t$ moves along the real axis of $t\left(t_{2}=0\right)$ from $t_{1}=-\infty$ to $t_{1}=-1$, thence around the upper semicircle of radius 1 , center at origin to the point $t_{1}=+1, t_{2}=0$, thence to $t_{1}=+\infty$, and finally returning to the starting point by way of a semicircle of infinite radius (center at the origin). This description of the path should be qualified by the statement that the branch points $t= \pm 1$ are avoided by means of small quarter circles, and the singular points $t=e^{i \alpha}$ and $t=\beta$, by means of semicircles as shown in Figure 2.

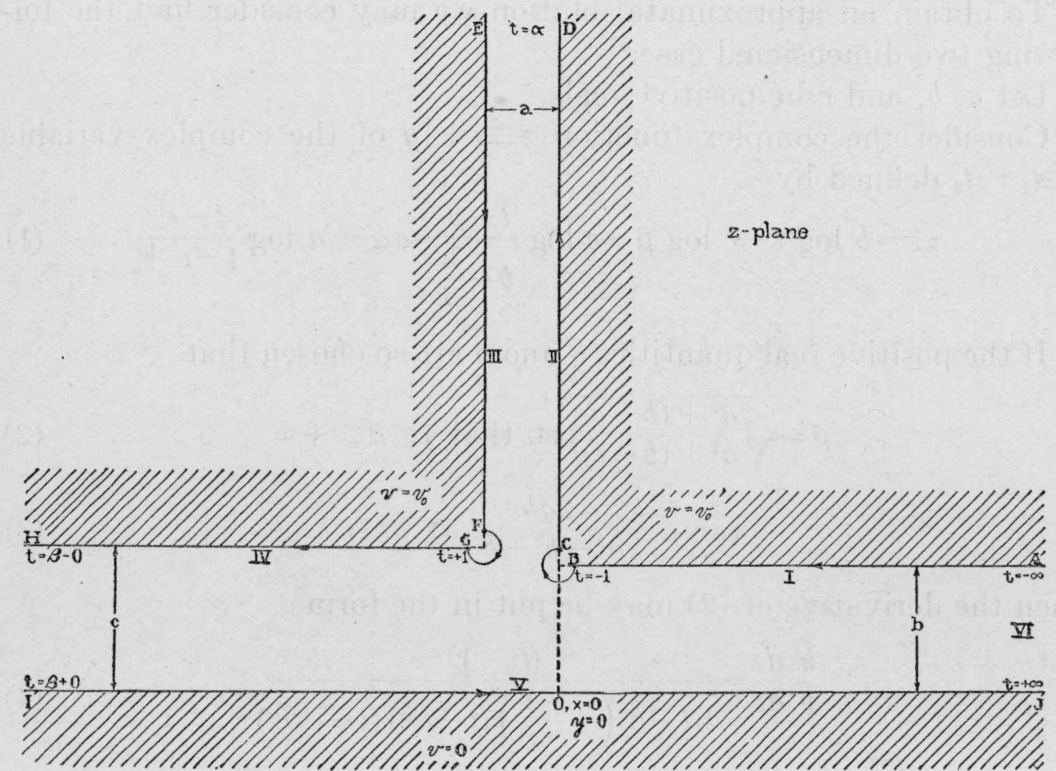

FIG. 1.-Conducting section in the z-plane

To verify the foregoing statement we may consider the six cases into which this description of the path naturally falls. If we replace $z$ by $x+i y$ and $t$ by $t_{1}+i t_{2}$ in (1) and equate reals and imaginaries, there result the two following equations:

$$
\begin{gathered}
\pi x=\frac{b}{2} \log \left(t_{1}^{2}+\dot{t}_{1}^{2}\right)-c \log \beta+\frac{c}{2} \log \frac{\left(t_{1}-\beta\right)^{2}+t_{2}^{2}}{\left(t_{1}-\frac{1}{\beta}\right)^{2}+t_{2}^{2}} \\
-a \alpha+a\left\{\tan ^{-1}\left(\frac{t_{2}-\sin \alpha}{t_{1}-\cos \alpha}\right)-\tan ^{-1}\left(\frac{t_{2}+\sin \alpha}{t_{1}-\cos \alpha}\right)\right\} \\
\pi y=b \tan ^{-1}\left(\frac{t_{2}}{t_{1}}\right)+c\left\{\tan ^{-1}\left(\frac{t_{2}}{t_{1}-\beta}\right)-\tan ^{-1}\left(\frac{t_{2}}{t_{1}-\frac{1}{\beta}}\right)\right\} \\
-\frac{a}{2} \log \frac{\left(t_{1}-\cos \alpha\right)^{2}+\left(t_{2}-\sin \alpha\right)^{2}}{\left(t_{1}-\cos \alpha\right)^{2}+\left(t_{2}+\sin \alpha\right)^{2}}
\end{gathered}
$$


If the point $t$ describes the contour $A B C D E F G H I J K A$ of Figure 2, the equations (6) take the following special forms in the six cases:

Case I. $-\infty<t_{1}<-1, t_{2}=0$

$$
\begin{gathered}
\pi x=b \log \left(-t_{1}\right)+c \log \frac{\beta-t_{1}}{1-\beta t_{1}}+2 a\left\{\pi-\frac{\alpha}{2}-\tan ^{-1}\left(\frac{\sin \alpha}{t_{1}-\cos \alpha}\right)\right\} \\
y=b \quad 0 \leqq x \leqq+\infty, \pi-\frac{\alpha}{2}<\tan ^{-1}\left(\frac{\sin \alpha}{t_{1}-\cos \alpha}\right) \leqq \pi
\end{gathered}
$$

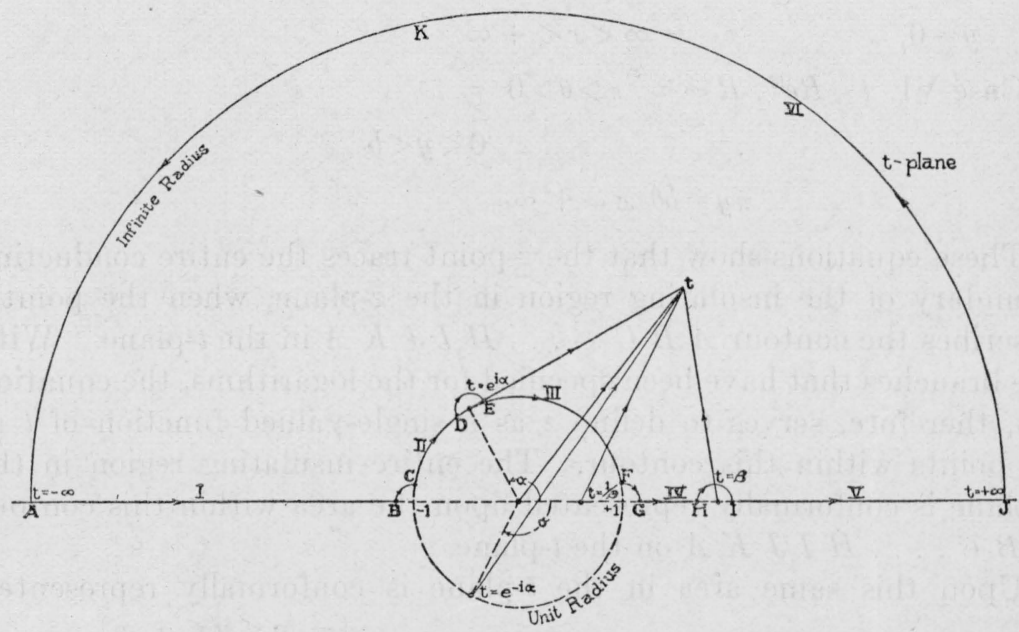

FIG. 2.-Corresponding region in the t-plane

Case II. $t=e^{\mathrm{i} \theta}$ or $\left\{\begin{array}{l}t_{1}=\cos \theta \\ t_{2}=\sin \theta\end{array}\right\} \alpha<\theta<\pi$

$$
\pi y=b \theta+c\left\{\tan ^{-1}\left(\frac{\sin \theta}{\cos \theta-\beta}\right)-\tan ^{-1}\left(\frac{\sin \theta}{\cos \theta-\frac{1}{\beta}}\right)\right\}-a \log \frac{\sin \left(\frac{\theta-\alpha}{2}\right)}{\sin \left(\frac{\theta+\alpha}{2}\right)}
$$

$$
b \leqq y \leqq+\infty
$$

Case III. Same as II except $\alpha>\theta>0$

$$
\begin{aligned}
\pi= & =b \theta+c\left\{\tan ^{-1}\left(\frac{\sin \theta}{\cos \theta-\beta}\right)-\tan ^{-1}\left(\frac{\sin \theta}{\cos \theta-\frac{1}{\beta}}\right)\right\}-a \log \frac{\sin \left(\frac{\alpha-\theta}{2}\right)}{\sin \left(\frac{\alpha+\theta}{2}\right)} \\
& c \leqq y \leqq+\infty
\end{aligned}
$$


Case IV. $1<t_{1}<\beta, t_{2}=0$

$$
\begin{aligned}
& \pi x=b \log t_{1}-c \log \beta+c \log \left(\frac{\beta-t_{1}}{t_{1}-\frac{1}{\beta}}\right)-2 a\left\{\tan ^{-1}\left(\frac{\sin \alpha}{t_{1}-\cos \alpha}\right)+\frac{\alpha}{2}\right\} \\
& y=c, \quad-\infty \leqq x \leqq-a
\end{aligned}
$$

Case V. $\beta<t_{1}<+\infty, t_{2}=0$

$$
\begin{aligned}
& \pi x=b \log t_{1}-c \log \beta+c \log \left(\frac{t_{1}-\beta}{t_{1}-\frac{1}{\beta}}\right)-2 a\left\{\tan ^{-1}\left(\frac{\sin \alpha}{t_{1}-\cos \alpha}\right)+\frac{\alpha}{2}\right\} \\
& y=0, \quad-\infty<x<+\infty
\end{aligned}
$$

Case VI. $t=R e^{i \theta}, R=\infty, \pi>\theta>0$

$$
\pi y=b \theta \quad x=+\infty \quad 0<y<b
$$

These equations show that the $z$-point traces the entire conducting boundary of the insulating region in the $z$-plane, when the point $t$ describes the contour $A B C \ldots \ldots H I J K A$ in the $t$-plane. With the branches that have been specified for the logarithms, the equation (1), therefore, serves to define $z$ as a single-valued function of $t$ at all points within this contour. The entire insulating region in the z-plane is conformally represented upon the area within this contour $A B C \ldots H I J K A$ on the $t$-plane.

Upon this same area in the $t$-plane is conformally represented an infinite strip of the $w$-plane, namely, $\left\{\begin{array}{c}-\infty<u<+\infty \\ 0<v<v_{0}\end{array}\right\}$, where $w$ is defined by (5). To show this one may replace $t$ by $t_{1}+i t_{2}$ in (5), and upon equating reals and imaginaries the two following equations are obtained:

$$
\begin{aligned}
& u=\frac{v_{0}}{\pi}\left\{-\frac{1}{2} \log \left(t_{1}{ }^{2}+t_{2}{ }^{2}\right)+\log \frac{\beta}{(\beta+1)^{2}}+\frac{1}{2} \log \left[\left(t_{1}-\beta\right)^{2}+t_{2}{ }^{2}\right]\right. \\
& +\frac{1}{2} \log \left[\left(t_{1}-\frac{1}{\beta}\right)^{2}+t_{2}^{2}\right] \\
& v=\frac{v_{0}}{\pi}\left\{-\tan ^{-1}\left(\frac{t_{2}}{t_{1}}\right)+\tan ^{-1}\left(\frac{t_{2}}{t_{1}-\beta}\right)+\tan ^{-1}\left(\frac{t_{2}}{t_{1}-\frac{1}{\beta}}\right)\right\}
\end{aligned}
$$

As special cases of these equations one finds the following expressions for $u$ and $v$ in the six cases previously considered:

Case I.

$$
\begin{aligned}
& u=\frac{v_{0}}{\pi} \log \left[\frac{\left(\beta-t_{1}\right)\left(\frac{1}{\beta}-t_{1}\right)}{-t_{1}} \cdot \frac{\beta}{(\beta+1)^{2}}\right] \\
& v=v_{0}
\end{aligned}
$$


Cases II and III.

$$
\begin{aligned}
& u=\frac{v_{0}}{\pi} \log \left[\frac{1-2 \beta \cos \theta+\beta^{2}}{(\beta+1)^{2}}\right] \\
& v=v_{0}
\end{aligned}
$$

Case IV.

$$
\begin{aligned}
& u=\frac{v_{\mathrm{o}}}{\pi} \log \left[\frac{\left(\beta-t_{1}\right)\left(t_{1}-\frac{1}{\beta}\right)}{t_{1}} \cdot \frac{\beta}{(\beta+1)^{2}}\right] \\
& v=v_{\mathrm{o}}
\end{aligned}
$$

Case V.

Case VI.

$$
\begin{aligned}
& u=\frac{v_{0}}{\pi} \log \left[\frac{\left(t_{1}-\beta\right)\left(t_{1}-\frac{1}{\beta}\right)}{t_{1}} \cdot \frac{\beta}{(\beta+1)^{2}}\right] \\
& v=0
\end{aligned}
$$

$$
\begin{aligned}
& u=\infty \\
& v=\frac{v_{0}}{\pi} \theta=v_{\mathrm{o}} \frac{y}{b}
\end{aligned}
$$

Hence, at all points within the contour $A B C \ldots H I J K A$ in the $t$-plane, $z$ and $w$ are each defined by (1) and (5), respectively, as single-valued functions of the complex variable $t$. If it were possible to eliminate $t$ between (1) and (5) and to obtain thereby an explicit formula for $w$ in terms of $z$, then upon equating imaginary components of this equation one would obtain an explicit expression for the potential $v$ at any point $x, y$, in the insulating region of Figure 1 , which potential vanishes at the lower conductor and assumes the constant value $v_{\mathrm{o}}$ upon each of the upper ones. It is harmonic at all points within the insulating region.

It is not possible to make such an elimination of $t$, although it is easy, by eliminating $t$, to obtain $z$ as a function of $w$. It is more convenient, however, to recognize in the equations (1) and (5) the solution of the electrostatic problem in the parametric form, and to retain the complex parameter $t$ in making all computations of density of electricity and ponderomotive force.

The upper right-hand conductor will be regarded (with certain modifications) as the attracted disk of an electrometer, the upper left conductor its guard ring, and the lower conductor the adjustable plate. The principal value of interest is the downward pull upon the disk and to obtain this one need only find the distribution of surface density of electricity upon its horizontal face; that is, along the line $B A$ which is Case I where

$$
-\infty<t_{1}<-1, t_{2}=0, v=v_{0}, y=b \text { and }\left\{\begin{array}{l}
0<x<+\infty \\
0<u<+\infty
\end{array}\right\}
$$


Case I gives

$$
\pi x=b \log \left(-t_{1}\right)+c \log \left(\frac{\beta-t_{1}}{1-\beta t_{1}}\right)-2 a\left\{\tan ^{-1}\left(\frac{-t_{1}-1}{-t_{1}+1} \cdot \tan \frac{\alpha}{2}\right)\right\}
$$

where $\tan ^{-1}\left(\frac{-t_{1}-1}{-t_{1}+1} \tan \frac{\alpha}{2}\right)$ ranges from zero to $\frac{\alpha}{2}$ as $t_{1}$ ranges from -1 to $-\infty$. Also

$$
u=\frac{\mathrm{v}_{\mathrm{o}}}{\pi} \log \left[\frac{\left(\beta=t_{1}\right)\left(1-\beta t_{1}\right)}{-t_{1}(\beta+1)^{2}}\right]
$$

The surface density of electricity, $\sigma$, at a value $t_{1}$ corresponding to the value $x$ is given by

$$
\begin{gathered}
4 \pi \sigma=\frac{\delta v}{\delta y}=\frac{\delta u}{\delta x}=\frac{d u}{d t_{1}} / \frac{d x}{d t_{1}}=\frac{d w}{d t_{1}} / \frac{d z}{d t_{1}} \\
=\frac{v_{0}}{\pi}\left\{\frac{t_{1}^{2}-1}{\mid t_{1}\left(t_{1}-\beta\right)\left(t_{1}-\frac{1}{\beta}\right)}\right) / \frac{b}{\pi} \frac{\left(t_{1}{ }^{2}-1\right)^{2}}{t_{1}\left(t_{1}-\beta\right)\left(t_{1}-\frac{1}{\beta}\right)\left(t_{1}-e^{\mathrm{i} \alpha}\right)\left(t_{1}-e^{-\mathrm{i} \alpha}\right)}
\end{gathered}
$$

by (4) and (5)

This gives$$
\sigma=\frac{v_{0}}{4 \pi b} \frac{\left(t_{1}-e^{\mathrm{i} \alpha}\right)\left(t_{1}-e^{-\mathrm{i} \alpha}\right)}{t_{1}^{2}-1}=\frac{v_{0}}{4 \pi b} \frac{t_{1}{ }^{2}-2 t_{1} \cos \alpha+1}{t_{1}{ }^{2}-1}
$$

and by (8), (9), and (10)

$$
\sigma d x=\frac{1}{4 \pi} d u
$$

Similarly one finds from (8), (9), and (10) that

$$
\begin{aligned}
2 \pi \sigma^{2} d x & =2 \pi \sigma^{2} d t \cdot \frac{d x}{d t} \\
& =2 \pi\left(\frac{v_{0}}{4 \pi b}\right)^{2}\left\{\frac{\left(t_{1}-e^{\mathrm{i} \alpha}\right)\left(t_{1}-e^{-\mathrm{i} \alpha}\right)}{t_{1}^{2}-1}\right\}^{2} \\
& \left\{\frac{b}{\pi} \cdot \frac{\left(t_{1}^{2}-1\right)^{2}}{t_{1}\left(t_{1}-\beta\right)\left(t_{1}-\frac{1}{\beta}\right)\left(t_{1}-e^{\mathrm{i} \alpha}\right)\left(t_{1}-e^{-\mathrm{i} \alpha}\right)}\right\} d t_{1} \\
& =2 \pi\left(\frac{v_{0}}{4 \pi b}\right)^{2} \frac{b}{\pi}\left\{\frac{\left(t_{1}-e^{\mathrm{i} \alpha}\right)\left(t_{1}-e^{-\mathrm{i} \alpha}\right)}{t_{1}\left(t_{1}-\beta\right)\left(t_{1}-\frac{1}{\beta}\right)}\right\} d t_{1} \\
& =2 \pi\left(\frac{v_{0}}{4 \pi b}\right)^{2} \frac{b}{\pi} d t_{1}\left\{\frac{1}{t_{1}}+\frac{\beta^{2}-2 \beta \cos \alpha+1}{\beta^{2}-1} \cdot\left[\frac{1}{t_{1}-\beta}-\frac{1}{\left(t_{1}-\frac{1}{\beta}\right)}\right]\right\}
\end{aligned}
$$

But from (2) and (3) it follows that

$$
\frac{\beta^{2}-2 \beta \cos \alpha+1}{\beta^{2}-1}=\frac{b}{c}
$$


Hence, the above relation becomes

$$
2 \pi \sigma^{2} d x=2 \pi\left(\frac{v_{0}}{4 \pi b}\right)^{2}\left\{\frac{b}{\pi t_{1}}+\frac{b^{2}}{\pi c}\left(\frac{1}{t_{1}-\beta}-\frac{1}{t_{1}-\frac{1}{\beta}}\right)\right\} d t_{1}
$$

from which it follows by integration that

$$
2 \pi \int_{0}^{x} \sigma^{2}\left(x_{1}\right) d x_{1}=2 \pi\left(\frac{v_{0}}{4 \pi b}\right)^{2}\left\{\frac{b}{\pi} \log \left(-t_{1}\right)+\frac{b^{2}}{\pi c} \log \left(\frac{\beta-t_{1}}{1-\beta t_{1}}\right)\right\}
$$

By elimination of the term $\log \frac{\beta-t_{1}}{1-\beta t_{1}}$ between this equation and (8) there results the equation

$$
\begin{gathered}
2 \pi \int_{0}^{x} \sigma^{2}\left(x_{1}\right) d x_{1}=2 \pi\left(\frac{v_{0}}{4 \pi c}\right)^{2}\left\{x+\frac{c^{2}-b^{2}}{\pi b} \log \left(-t_{1}\right)\right. \\
\left.+\frac{2 a}{\pi} \tan ^{-1}\left(\frac{-t_{1}-1}{-t_{1}+1} \tan \frac{\alpha}{2}\right)\right\}
\end{gathered}
$$

The parametric equations (8) and (10) with parameter $t_{1}$ define the surface density $\sigma$ as a function of $x$. They show that $\sigma$ becomes infinite when $x$ approaches zero, and in such a manner that the principal part of $\sigma$ is proportional to $x^{-\frac{1}{3}}$. In fact, when $x$ is small one obtains the expression

$$
\sigma=\frac{v_{0}}{4 \pi b}(1+\cos \alpha)\left\{\left(\frac{a}{h x}\right)^{\frac{1}{3}}+\frac{1+\left(\frac{h x}{a}\right)^{\frac{1}{3}}}{2+\left(\frac{h x}{a}\right)^{\frac{3}{3}}}\right\}
$$

in which terms of the order of $x^{4 / 3}$ are neglected. The positive quantity $h$ occurring in this formula is defined by

$$
h=3 \pi(1+\cos \alpha)\left\{\sin \alpha+\frac{a}{b}(1+\cos \alpha)\right\}
$$

The equations (16) and (17) may be obtained by noting that according to (8) and (10) the positive quantities $x$ and $\tau \equiv-1-t_{1}$ vanish together. The equation (8) may be put in the form

$$
\begin{aligned}
\pi x & =b \log (1+\tau)+c \log \left(\frac{1+\frac{\tau}{\beta+1}}{1+\frac{\beta \tau}{\beta+1}}\right)-i a \log \left(\frac{1+\frac{\tau e^{-i \frac{\alpha}{2}}}{2 \cos \frac{\alpha}{2}}}{1+\frac{\tau e^{i \frac{\alpha}{2}}}{2 \cos \frac{\alpha}{2}}}\right) \\
& =b \sum_{n=1}^{\infty} \frac{(-1)^{\mathrm{n}} \tau^{\mathrm{n}}}{n}\left\{-1+\frac{c}{b} \frac{\beta^{\mathrm{n}-1}}{(\beta+1)^{\mathrm{n}}}+2 \frac{a}{b} \frac{\sin \frac{n \alpha}{2}}{2^{\mathrm{n}} \cos \frac{n \alpha}{2}}\right\}
\end{aligned}
$$


This series converges if $0<\tau<2 \cos \frac{\alpha}{2}$. The coefficient of $\tau^{\mathrm{n}}$ vanishes identically for $n=1$ and for $n=2$ since it is found from (2) and (3) that

$$
-1+\frac{c}{b} \frac{\beta-1}{\beta+1}+\frac{a}{b} \frac{\sin \alpha}{1+\cos \alpha}=0
$$

Consequently, the series (18) begins with the term $n=3$ so that if $\tau^{4}$ be neglected (that is, $x^{4 / 3}$ ) one may write

$$
\frac{h x}{a}=\tau^{3} \text { or } \tau=\left(\frac{h x}{a}\right)^{\frac{1}{3}}
$$

where $h$ is defined by (17).

The equation (10) becomes in terms of $\tau$

$$
\sigma=\frac{v_{\mathrm{o}}}{4 \pi b}(1+\cos \alpha)\left\{\frac{1}{\tau}+\frac{1+\frac{\tau}{1+\cos \alpha}}{2+\tau}\right\}
$$

so that if the value of $\tau$ from (20) be used in (21) the result is equation (16).

When $a$ and $c-b$ are both small compared to $b$, the equations (8) and (10) show that the density $\sigma$ very rapidly approaches its final value $\frac{v_{0}}{4 \pi b}$ when $x$ takes on an appreciable value.

Returning again to the consideration of (15) it is evident that when $a$ and $c-b$ are both small compared to $b$ and to $x$ (although the ratio $\frac{x}{b}$ may have any value) those terms in which $t_{1}$ appears in the second member of (15) are small correction terms. In the latter, $t_{1}$ may, therefore, be replaced by an approximate expression in terms of $x$ thus leading to an explicit expression in $x$ in place of the second member of (15). To obtain such an approximate expression we may note that, according to (2), $\frac{1}{\beta}$ is a small positive value, namely,

$$
\frac{1}{\beta}=\frac{\rho}{\sqrt{(b+c)^{2}+a^{2}}}=\frac{\rho}{2 c} \text { (to the first order) }
$$

where

$$
\rho=\sqrt{(c-b)^{2}+a^{2}}
$$

If (8) be rewritten in the form

$$
\begin{gathered}
\frac{\pi x}{c}+\log \beta=\log \frac{\left(\beta-t_{1}\right)\left(-t_{1}\right)}{\frac{1}{\beta}-t_{1}}-\frac{c-b}{c} \log \left(-t_{1}\right) \\
-\frac{2 a}{c} \tan ^{1}-\left(\frac{-t_{1}-1}{-t_{1}+1} \tan \frac{\alpha}{2}\right)
\end{gathered}
$$


then it is evident that the last two terms, which contain the small factors $\frac{c-b}{c}$ and $\frac{a}{c}$, may be neglected and this leads to the approximate equation (when neither $x$ nor $t_{1}+1$ are small)

$$
\frac{\pi x}{c}+\log \beta=\log \frac{\left(\beta-t_{1}\right)\left(-t_{1}\right)}{\frac{1}{\beta}-t_{1}}
$$

or

$$
\frac{\left(\beta-t_{1}\right)\left(-t_{1}\right)}{\frac{1}{\beta}-t_{1}}=\beta e^{\frac{\pi x}{6}}
$$

which upon being solved for $t_{1}$ gives

$$
\begin{gathered}
-t_{1}=\frac{\beta}{2}\left(e^{\frac{\pi x}{c}}-1\right)+\sqrt{\frac{\beta^{2}}{4}\left(e^{\frac{\pi x}{e}}-1\right)^{2}+e^{\frac{\pi x}{c}}} \\
=\beta\left(e^{\frac{\pi x}{c}}-1\right)
\end{gathered}
$$

(very approximately)

or

$$
-t_{1}=\frac{2 b}{\rho}\left(e^{\frac{\pi \mathrm{x}}{\mathrm{c}}}-1\right)
$$

This approximate value of $t_{1}$ is valid whatever value the ratio $\frac{x}{c}$ may have so long as both $x$ and $c$ are large compared to $a$ and to $c-b$, which is always the condition in the applications to be made.

Replacing $-t_{1}$ in the second member of (15) by the value (25) gives

$$
\begin{gathered}
2 \pi \int_{0}^{x} \sigma^{2}\left(x_{1}\right) d x_{1}=2 \pi\left(\frac{v_{0}}{4 \pi c}\right)^{2}\left\{x+\frac{c^{2}-b^{2}}{\pi b} \log \frac{2 c}{\rho}\left(e^{\frac{\pi \mathrm{x}}{\mathrm{c}}}-1\right)\right. \\
\left.+\frac{2 a}{\pi} \tan ^{-1}\left(\frac{2 c\left(e^{\frac{\pi \mathrm{x}}{\mathrm{c}}}-1\right)-\rho}{2 c\left(e^{\frac{\pi \mathrm{X}}{\mathrm{c}}}-1\right)+\rho} \tan \frac{\alpha}{2}\right)\right\}
\end{gathered}
$$

The last term may be replaced by $\frac{a \alpha}{\pi}$ for all cases that arise in practice.

This is evident since $\pm \rho$ is negligible compared to $2 c\left(e^{\frac{\pi x}{c}}-1\right)$. Its retention would simply give a correction to the small correction term and perhaps would not be justifiable considering the approximations already made. It is easy to see that the term $2 c\left(e^{\frac{\pi x}{c}}-1\right)$ is large compared to $\rho$ even in the most unfavorable case where $\frac{x}{c}$ is very small 
(although $x$ is large compared to $a$ or $c-b$ ). In this case it reduces to

$$
2 c\left\{1-1+\frac{\pi x}{c}+\frac{1}{2}\left(\frac{\pi x}{c}\right)^{2}+\cdots \cdot\right\}=2 \pi x \text { (approximately) }
$$

Consequently,

$$
2 \tan ^{-1}\left(\frac{2 c\left(e^{\frac{\pi x}{c}}-1\right)-\rho}{2 c\left(e^{\frac{\pi x}{c}}-1\right)+\rho} \tan \frac{\alpha}{2}\right)=\alpha=\tan ^{-1} \frac{a}{c-b} \text { (approximately) }
$$

The equation (26) may finally be written in the following form

where

$$
\begin{aligned}
2 \pi \int_{0}^{x} \sigma^{2}\left(x_{1}\right) d x_{1}=2 & \left(\frac{v_{0}}{4 \pi c}\right)^{2}\left\{x+\frac{2(c-b)}{\pi} \log \frac{2 c\left(e^{\frac{\pi x}{c}}-1\right)}{\sqrt{(c-b)^{2}+a^{2}}}\right. \\
& \left.+\frac{a}{\pi} \tan ^{-1}\left(\frac{a}{c-b}\right)\right\}
\end{aligned}
$$

$$
0<\tan ^{-1} \frac{a}{c-b}<\pi
$$

The physical meaning of (27) is to be found in the fact that the force which acts downward upon unit area of the conductor is $2 \pi \sigma^{2}$. Hence, the force acting upon a rectangle of length $l$ (perpendicular .to the paper in fig. 1) and breadth $x$, whose area is $S=l x$, is therefore

$$
\begin{aligned}
F_{1} & =2 \pi l \int_{0}^{\mathrm{x}} \sigma^{2}\left(x_{1}\right) d x_{1} \\
& =2 \pi\left(\frac{v_{\mathrm{o}}}{4 \pi c}\right)^{2}\left\{S+\frac{2 l(c-b)}{\pi} \log \frac{2 c\left(e^{\frac{\pi x}{c}}-1\right)}{\sqrt{(c-b)^{2}+a^{2}}}+\frac{l a}{\pi} \tan ^{-1}\left(\frac{a}{c-b}\right)\right\}
\end{aligned}
$$

The principal term in the second member of (28) is $2 \pi\left(\frac{v_{0}}{4 \pi c}\right)^{2} S$; that is, the force, computed upon the assumption of a constant density $\left(\frac{v_{0}}{4 \pi c}\right)$. The remaining terms, which are multiplied by $l$, represent the small correction due to the nonuniformity of density along the edge $x=0$, whose length is $l$.

With this interpretation in mind, we may now make application to the case of a circular attracted disk which is separated from its guard plane by a small horizontal distance $a$ and displaced downward a small distance $c-b$ where $c$ is the separation between the guard plane and the plate at zero potential.

If the radius of the disk be $x$ then its area $S$ is $\pi x^{2}$ and its perimeter $l$ is $2 \pi x$, and if this is large compared to $a$ and to $b-c$ the edge correction will be that represented by terms containing the factor $l$ in the above 
equation. Using $r$ for $x$ (the radius of the disk) we obtain, therefore, the following approximate expression for the force upon the attracted disk:

$$
\begin{aligned}
F_{1}=\frac{v_{0}^{2} r^{2}}{8 c^{2}}\left\{1+\frac{a}{r}-\right. & \frac{2}{\pi}\left[\frac{a}{r} \tan ^{-1} \frac{c-b}{a}+\frac{c-b}{r} \log \left((c-b)^{2}+a^{2}\right)\right] \\
+ & \left.\frac{4(c-b)}{\pi r} \log 2 c\left(e^{\frac{\pi \mathrm{r}}{\mathrm{c}}}-1\right)\right\}
\end{aligned}
$$

provided $a$ and $c-b$ are each small compared to $r$ or to $c$. (The ratio of $r$ to $c$ may then have any value.) In this formula

$$
-\frac{\pi}{2}<\tan ^{-1}\left(\frac{c-b}{a}\right)<\frac{\pi}{2}
$$

and the radius of the attracted disk is $r$, its height above the zeropotential plate is $b$, its clearance from its guard plate is $a$, and the height of the latter above the plate of zero potential is $c$. The second member involves only the ratios of these lengths and will, therefore, be unaffected by the choice of unit of length. The potential of the attracted disk and its guard plane is $v_{0}$ electrostatic c. g. s. units, and the total downward force $\mathrm{F}_{1}$ which acts upon the attracted disk is measured in dynes. The distribution of electricity upon the sides of the attracted disk contributes nothing to the downward force since the field is horizontal at these sides. The disk and its guard plate have been assumed to be infinitely thick, which means that they are so thick that no appreciable electrification appears upon their upper sides. This is evidently a good approximation for a disk $5 \mathrm{~cm}$ in radius and $1 \mathrm{~cm}$ in thickness when the aperture $a$ is less than $1 / 20 \mathrm{~cm}$, which is approximately the case for the absolute electrometer under construction at this bureau.

Since the equation (24) showing how the force $F$ acting upon the attracted disk depends upon the clearance $a$ between it and its guard plane and upon its displacement $c-b$ below the plane of the latter has been obtained on the assumption that these distances are each small compared to the radius of the disk, or to the separation of the planes $c$ or $b$, it is evident this equation still holds (and with increasing precision) when this separation is made very large. A separation in fact of $110 \mathrm{~cm}$ is contemplated in the use of the absolute electrometer. It may be noted that the value of the first correction terms in (29), namely,

$$
\frac{a}{r}-\frac{2}{\pi}\left[\frac{a}{r} \tan ^{-1}\left(\frac{c-b}{a}\right)+\frac{c-b}{r} \log \left((c-b)^{2}+a^{2}\right)\right]
$$

is not dependent upon this separation since $(c-b)^{2}+a^{2}$ is the square of the length of the line from the edge of the disk to the edge of the guard ring and $\tan ^{-1}\left(\frac{a}{c-b}\right)$ is the angle which this line makes with 
the horizontal. In case the disk is accurately adjusted to the plane of its guard ring this term reduces to $\frac{a}{r}$.

The last correction term is only important when the disk is displaced. This term depends upon the displacement $c-b$ and also upon the distance $c$ of the lower plate, and if the latter is fairly close (say $1 \mathrm{~cm}$ while $r=5 \mathrm{~cm}$ ) then $e^{+\frac{\pi \mathrm{r}}{\mathrm{c}}}=e^{+15}$ which is large compared to 1 so that this correction term reduces to $\frac{4(c-b)}{\pi r}\left(\frac{\pi r}{c}+\log 2 c\right)$. On the other hand, when the lower plate of zero potential is lowered to a great depth $e^{\frac{\pi \mathrm{r}}{\mathrm{c}}}-1$ becomes very small and $2 c\left(e^{\frac{\pi \mathrm{r}}{\mathrm{c}}}-1\right)$ approaches

$$
2 c\left[1-1+\frac{\pi r}{c}+\frac{1}{2}\left(\frac{\pi r}{c}\right)^{2}+\cdots\right]
$$

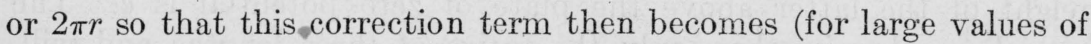
$c$ compared to $\pi r$ )

$$
\frac{4(c-b)}{\pi r} \log 2 \pi r
$$

If it is possible to adjust the position of the disk so accurately that the correction terms due to its displacement $(c-b)$ are negligible then the force upon it may be computed by the formula

$$
F_{1}=\frac{v_{0}{ }^{2} r^{2}}{8 b^{2}}\left(1+\frac{a}{r}\right)=\frac{v_{0}{ }^{2}\left(r+\frac{a}{2}\right)^{2}}{8 b^{2}}
$$

which shows that for this case the effective radius of the disk is the arithmetic mean of the inner and outer radii of the gap. However, it must be noted that this assumes that $c-b$ may be made very small indeed, for the principal correction term is

$$
\frac{4}{\pi} \cdot \frac{c-b}{r} \log \frac{2 c\left(e^{\frac{\pi \mathrm{r}}{c}}-1\right)}{\sqrt{(c-b)^{2}+a^{2}}}
$$

Even if $c-b$ is small compared to $a$ this becomes

$$
\frac{4}{\pi} \cdot \frac{c-b}{r} \log \frac{2 c}{a}\left(e^{\frac{\pi \mathrm{r}}{\mathrm{c}}}-1\right)
$$

which may be rather large and which has the same algebraic sign as $c-b$. This means that when the disk is displaced dowuward the downward force increases. Consequently, this term is to be considered in estimating the amount by which the application of the potential decreases the stability of the system when the disk is hung from one arm of a balance. 
III. EFFECT OF THE CIRCULAR CONDUCTING HOOPS WHICH ARE USED TO INCREASE THE UNIFORMITY OF FIELD AT THE DISK

Since it is desired to work with separation of the plates $b$, which is of the same order of magnitude as their diameter $2 R$, a number, $N$, of conducting hoops of radius approximately equal to $R$ are used to increase the uniformity of field in the neighborhood of the disk, which is situated centrally in the upper plate and has a radius $r$ of about $5 \mathrm{~cm}$, whereas $R$ is about $50 \mathrm{~cm}$. A hoop whose height is $y$ above the lower plate, is kept at a potential $v=\frac{y}{b} v_{\text {o }}$ above that

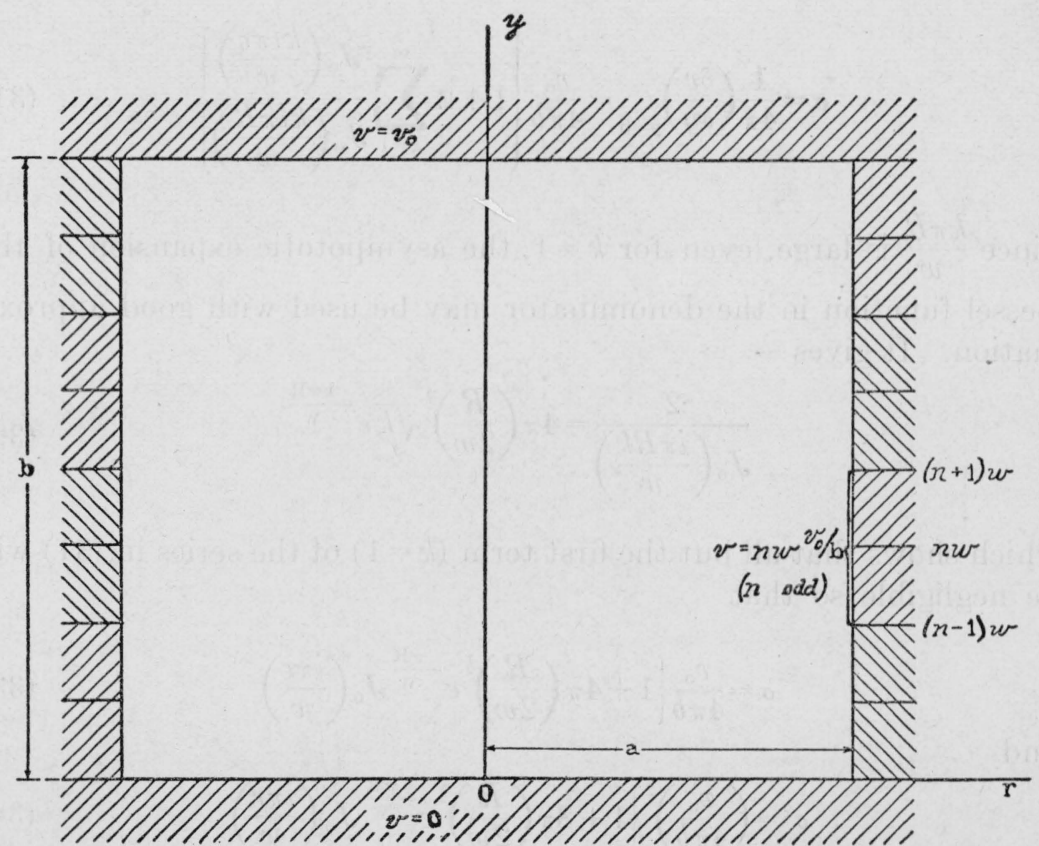

Fig. 3.-Principal section of cylinder

plate, where $v_{0}$ is the amount by which the upper plate exceeds the lower in potential. To find the effect of these hoops upon the force acting upon the attracted disk, the conditions may be idealized as follows:

Instead of hoops, it will be assumed that the cylindrical surface $r=R$, between $y=0$ and $y=b$, is divided into $N$ equal bands each of width $2 w$ over which the potential is constant. Thus if $n$ is an odd integer, $1,3,5, \ldots \ldots$ the band for which $(n-1) w<y<(n+1) w$ has the constant potential $n w \frac{v_{0}}{b}$. The potential function is

(1) Harmonic within the cylinder $0<r<R, 0<y<b$,

(2) Vanishes for $y=0$, 
(3) is equal to the constant $v_{0}$ for $y=b$, and,

(4) reduces on the convex surface $r=R$, to the discontinuous function of $y$ described above.

It may be constructed by ordinary methods and is found to be

$$
v=\frac{v_{\mathrm{o}}}{b}\left\{y+\frac{2 w}{\pi} \sum_{k=1}^{\infty} \frac{\sin \frac{k \pi y}{w}}{k} \frac{J_{\mathrm{o}}\left(\frac{i k \pi r}{w}\right)}{J_{\mathrm{o}}\left(\frac{i k \pi R}{w}\right)}\right\}
$$

where $J_{0}$ is the Bessel's function of zero order. This gives for the density of electricity on the upper plate, $y=b$, the value

$$
\boldsymbol{\sigma}=\frac{\mathbf{1}}{4 \pi}\left(\frac{\delta v}{\delta y}\right)_{\mathrm{y}=\mathrm{b}}=\frac{v_{0}}{4 \pi b}\left\{1+2 \sum_{k=1}^{\infty} \frac{J_{\mathrm{o}}\left(\frac{k i \pi r}{w}\right)}{J_{\mathrm{o}}\left(\frac{k i \pi R}{w}\right)}\right\}
$$

Since $\frac{k \pi R}{u}$ is large, even for $k=1$, the asympototic expansion of the Bessel function in the denominator may be used with good approximation. It gives

$$
\frac{2}{J_{\mathrm{o}}\left(\frac{i \pi R k}{w}\right)}=4 \pi\left(\frac{R}{2 w}\right)^{\frac{1}{2}} \sqrt{k} e^{-\frac{\mathrm{k} \pi \mathrm{R}}{\mathrm{w}}}
$$

which shows that all but the first term $(k=1)$ of the series in (31) will be negligible so that

and

$$
\sigma=\frac{v_{0}}{4 \pi b}\left\{1+4 \pi\left(\frac{R}{2 w}\right)^{\frac{1}{2}} e^{-\frac{\pi \mathrm{R}}{w}} J_{\mathrm{o}}\left(\frac{i \pi r}{w}\right)\right.
$$

$$
\sigma^{2}=\left(\frac{v_{0}}{4 \pi b}\right)^{2}\left\{1+8 \pi\left(\frac{R}{2 w}\right)^{\frac{1}{2}} e^{-\frac{\pi \mathrm{R}}{w}} J_{0}\left(\frac{i \pi r}{w}\right)\right.
$$

The total downward force upon a circular area in the plate $y=b$, whose center is on the axis of the cylinder and whose radius is $r$, is

$$
F=2 \pi \int_{0}^{\mathrm{r}} \sigma^{2} 2 \pi r d r=\frac{v_{0}^{2} r^{2}}{8 b^{2}}\left\{1+16 \pi\left(\frac{R}{2 w}\right)^{\frac{1}{2}} e^{-\frac{\pi \mathrm{R}}{\mathrm{w}}} \frac{J_{1}\left(\frac{i \pi r}{w}\right)}{\left(\frac{i \pi r}{w}\right)}\right\}
$$

where $J_{1}$ is the Bessel function of order one and may be written

$$
J_{1}\left(\frac{i \pi r}{w}\right)=\frac{i \pi r}{2 w} \sum_{s=0}^{\infty} \frac{\left(\frac{\pi r}{2 b}\right)^{2 \mathrm{~s}}}{s !(s+1) !}
$$


The equation (35), therefore, gives the correction term to the force on the attracted disk of radius $r$ due to the effect of the $2 N$ rings which are maintained at appropriate potentials. As a matter of fact $r$ is about $5 \mathrm{~cm}$ so that $\frac{\pi r}{w}$ is usually so large that one may also use the asymptotic expansion for $J_{1}$ in this equation. This gives

$$
F=\frac{v_{\mathrm{o}}^{2} r^{2}}{8 b^{2}}\left\{1+8\left(\frac{R}{r}\right)^{\frac{1}{2}} \frac{e^{\frac{-\pi(\mathrm{R}-\mathrm{r})}{\mathrm{w}}}}{\left(\frac{\pi r}{w}\right)}\right\}
$$

where $2 w$ is the distance between the hoops. Since the first term $\frac{v_{0}^{2} r^{2}}{8 b^{2}}$ is the force on a circular area of radius $a$ when both the planes $y=0$ and $y=b$ are infinite in extent, the correction terms $\Delta F$ due to their finiteness when shielded as described is just

$$
\Delta F=\frac{v_{0}^{2} r^{2}}{8 b^{2}}\left\{8\left(\frac{R}{r}\right)^{\frac{1}{2}} \frac{e^{\frac{-\pi(\mathrm{R}-\mathrm{r})}{\mathrm{w}}}}{\left(\frac{\pi r}{w}\right)}\right\}
$$

\section{SUMMARY}

The equations (29) of Section I and (38) of Section II may now be assembled to give in a single equation the corrections to the force, which are due to all the circumstances that have been considered. This gives for the downward force (in dynes) upon the attracted disk $F=F_{1}+\Delta F$ or

where

$$
\begin{gathered}
F=\frac{v_{0}^{2} r^{2}}{8 c^{2}}\left\{1+\frac{a}{r}-\frac{2 a}{\pi r} \tan ^{-1}\left(\frac{c-b}{a}\right)+\frac{4(c-b)}{\pi r} \log \frac{2 c\left(e^{\frac{\pi \mathrm{r}}{c}}-1\right)}{\sqrt{(c-b)^{2}+a^{2}}}\right. \\
\left.+8\left(\frac{R}{r}\right)^{\frac{1}{2}} \frac{e^{\frac{-\pi(\mathrm{R}-\mathrm{r})}{\mathrm{W}}}}{\left(\frac{\pi r}{w}\right)}\right\}
\end{gathered}
$$

$v_{0}=$ the difference of potential in electrostatic c. g. s. units,

$r=$ radius of the attracted disk,

$b=$ its height above the adjustable plate,

$c=$ the height of the guard plane above the plate,

$a=$ clearance between disk and guard plane,

$R=$ radius of adjustable plate and of guard plane,

$2 w=$ the distance between adjacent hoops of radius $R$ which are equally spaced between the plates and kept at potentials proportional to their distance from the lower plate. 
In deriving this formula it has been assumed that $a$ and $c-b$ are each small compared either to $r$ or $c$, although the ratio $\frac{r}{c}$ may have any value.

It has also been assumed in obtaining the last term in (38), namely,

$$
8\left(\frac{R}{r}\right)^{\frac{1}{2}} \frac{e^{\frac{-\pi(\mathrm{R}-\mathrm{r})}{\mathrm{w}}}}{\left(\frac{\pi r}{w}\right)}
$$

that $\frac{\pi r}{w}$ is so large that the first term of the asymptotic expansion of the Bessel's function in (35) is a sufficient approximation to that function. If this is not the case the general expression involving $J_{1}$ must be retained. However, this will never be necessary for the application here contemplated, for the asymptotic expansion here used for $J_{\mathrm{o}}(i x)$ is in error by less then one-third of 1 per cent even for values of $x$ as small as 10, as may be seen by reference to Jahnke und Emde's "Funktionentafeln Mit Formeln und Kurven," page 130. The smallest argument occurring here is in the case of $J_{\mathrm{o}}\left(\frac{i k \pi r}{w}\right)$ or $J_{0}\left(\frac{i \pi r}{w}\right)$ when $k=1$. With $r=5 \mathrm{~cm}$ and $w=1 \mathrm{~cm}, \frac{\pi r}{w}$ is $5 \pi$ or 15.7. For this and all higher values of the argument, the asymptotic expansions here used have a precision far beyond that attainable by experiment. A high order of precision in the last term with the bracket of equation (38) is not needed since this term is itself a very small correction term. In fact, if $w=1 \mathrm{~cm}, r=5 \mathrm{~cm}$, and $R=50 \mathrm{~cm}$, it reduces to $1.61 e^{-45 \pi}$, which shows that the arrangement of hoops will produce practically perfect compensation if they can be maintained at the desired potentials.

Washington, December 7, 1926. 